\title{
Impact of bilateral total salpingectomy versus standard tubal ligation at time of cesarean section on ovarian reserve: A randomized controlled trial
}

Original

Article
Mohamed Anwar Elnory, Ashraf Nassif Mahmoud Elmantwe

Department of Obstetrics and Gynecology, Faculty of Medicine, Benha University, Benha, Egypt

\begin{abstract}
Aim of the work: The objective of this trial was to compare between bilateral total salpingectomy (BTS) as a theoretically more effective ovarian cancer $(\mathrm{OvCa})$ risk-reducing primary prevention and bilateral partial salpingectomy (BPS) [stander tubal ligation (STL)] procedures at time of cesarean section (CS) for parturients who completed their reproductive desire and requesting permanent sterilization regard to the impacts of both procedures on ovarian reserve parameters at one year post-sterilization as well as the surgical consequences of both procedures.

Patients and Methods: This is a prospective, randomized, open-label, concealed allocation, parallel group, superiority trial was conducted at three surgical sites in El-Qulobia, Egypt including Benha University Hospital, Obstetrics and Gynecology Department between October 2015 and October 2017. 120 parturients undergoing CS were allocated to BTS (intervention group) (60) and BPS (control group) (60). Main outcomes were one year post-sterilization differences in ovarian reserve (OR) parameters including hormonal as well as two-dimensional and three-dimensional transvaginal ultrasonographic parameters ; namely, anti-müllerian hormone $(\mathrm{AMH})(\mathrm{ng} / \mathrm{ml})$, follicular stimulating hormone (FSH) $(\mathrm{mIu} / \mathrm{ml})$, peak systolic velocity (PSV) $(\mathrm{cm} / \mathrm{s})$, antral follicle count (AFC) (n), vascular index (VI) $(\%)$, flow index (FI) (0-100), vascular flow index (VFI) (0-100), calculated ovarian age [OvAge (year)] and ovarian volume. Ancillary outcomes were surgical feasibility and surgical consequences.

Results: 102 women completed the one-year follow up (85\%), 50 in the BTS group and 52 in the BPS group and there were no statistically significant differences regarding the ovarian reserve parameters at one year post-sterilization including AMH, FSH, AFC, VI, FI, VFI OvAge and ovarian volume $(P>0.05)$. Also, there were no statistically significant differences regarding items included in surgical. consequences comparison $(P>0.05)$ as well as surgical sterilization feasibility $(100 \%$ vs $100 \%, p=1)$. Also, there were no apparent complications due to sterilization procedures in both groups.

Conclusion: Impacts of bilateral total salpingectomies (BTS) on ovarian reserve as sterilizing procedure during cesarean section as well as rates of surgical consequences are similar with that of bilateral partial salpingectomies (BPS) (STL). So, BTS could be recommended over BPS (STL) for women completing their reproductive desire, despite its better efficacy as ovarian cancer reducing surgery as well as its better clinical and sterilizing efficacy remained to be elucidated. .
\end{abstract}

Key Words: Anti-müllerian hormone, cesarean section, ovarian cancer, ovarian reserve, sterilization, total salpingectomy, tubal ligation.

Received: 12 October 2018, Accepted: 13 February 2019

Corresponding Author: Ashraf Nassif Mahmoud Elmantwe, Department of Obstetrics and Gynecology, Benha University, Banha, Egypt, Tel.: 01001801244, E-mail: ashrafnassifelmantwe@fmed.bu.edu.eg

ISSN: 2090-7265, May 2019, Vol.9, No.2

\section{INTRODUCTION}

Ovarian cancer (OvCa) is the most frequent cause of gynecological cancer mortality and its lifetime risk is $\approx 1.4 \%$ in developed countries as well as accounted to be the fifth common cause of cancer mortality in women, despite the advances in its surgical, chemotherapeutic and radiological interventions ${ }^{[1]}$. Owing to these OvCa related issues as well as lack of OvCa effective screening programs and always late advanced presentation of $\mathrm{OVCa}^{[1]}$, in addition to new discoveries of fallopian tubes
(FTs) pivotal roles in tumorigenesis of both type I and type II epithelial ovarian carcinomas (EOCs $)^{[2,3,4,5,6]}$, the focus of decreasing EOCs' clinical, economical as well as social burden had been shifted towards the primary prevention throughout post-reproductive FTs removal in average-risk women for $\mathrm{OvCa}$, which is known as bilateral opportunistic salpingectomy (BOS) or risk-reducing salpingectomy (RRS) or bilateral prophylactic salpingectomy (BPS ${ }^{[7,8]}$.

Sterilization is performed in an estimated percentage of $19 \%$ of reproductive age women worldwide ${ }^{[9]}$. Both excisional [bilateral partial salpingectomy (BPS)] and 
non-excisional tubal ligations (TLs) have been associated with reduced OvCa risk, especially low-grade type I Sterilization is performed in an estimated percentage of $19 \%$ of reproductive age women worldwide ${ }^{[9]}$. Both excisional [bilateral partial salpingectomy (BPS)] and non-excisional tubal ligations (TLs) have been associated with reduced OvCa risk, especially low-grade type I endometrioid and clear cell $\mathrm{OvCa}$ due to interruptions of FTs' conduit connection between uterine cavity and lower genital tract containing dysplastic cells as well as carcinogenic agents to the ovarian environment ${ }^{[6]}$. Moreover, excisional TLs [including the commonly performed procedures BPS which coined as standard tubal ligation (STL)] and bilateral total salpingectomy (BTS) were found to add more OvCa risk reduction depending on the amount of FTs removed ${ }^{[6]}$. Ely and Truong in their meta-analysis deduced that BPS might reduce OvCa by 13 to $41 \%$ and BTS might decrease OvCa by $42 \%-78 \%{ }^{[10]}$. While, Yoon et al. in their meta-analysis showed that $\mathrm{BOS}$ at the of hysterectomy reduce the lifetime OvCa risk by $\approx 50 \%{ }^{[11]}$; but owing to low absolute OvCa risk, the number of women needed to be treated with BTS (NNT) to prevent one OvCa case is relatively high at 366 as well as the NNT to prevent on OvCa related mortality is also high at $450^{[12]}$.

BPS has the highest sterilizing efficacy compared with others TLs, as it has lowest cumulative pregnancy rate $7.5 / 1000^{[13]}$ and ectopic rate of $1.2 / 1000^{[14]}$ as well as its surgical profile relatively safe, especially at the time of cesarean section $(\mathrm{CS})^{[15,16]}$. While, BTS offers more theoretical sterilizing efficacy than BPS as well as eliminating risks of tubal ectopics, hydrosalpinges, pyosalpinges and Morgagni hydatids ${ }^{[12,16]}$. However, these BTS related potential benefits are offered at the expense of more surgical risks including mesosalpingial and ovarian vascular plexus tearing with subsequent intraabdominal hemorrhage, especially at postpartum (PP) tubal sterilization $(\mathrm{TS})^{[15,16]}$. These high surgical consequences with BTS are secondary to pregnancy-related pelvic engorgement and anatomical changes ${ }^{[15,16]}$. Trials comparing BPS versus BTS at Cs concentrated on surgical consequences evaluation $^{[17,18,19,20,21,22]}$, rather than impacts of this surgical procedures on ovarian blood supply and its subsequent impacts on ovarian functions ${ }^{[23]}$.

This work aimed to assess performed BTS versus BPS at the time of CS in women who were aiming in permanent sterilization regard to the detrimental impacts on ovarian vascularization, function and reserve, in addition to comparing procedures surgical profile safety and consequences.

\section{PATIENTS AND METHODS}

We conducted this open-label, parallel group, concealed allocated, randomized, controlled, superiority trial, prospectively at three surgical, obstetrical sites ; namely, Benha University Hospital (BUH), Obstetrics and Gynecology Department, El-Hayat Specialized Gynecology and Obstetrics Center at Benha City and ElTaqwa Surgical Center at Abu-Zabal, all these surgical sites were in El-Qalubia, Egypt. We conducted this trial between October 2015 and October 2017. Benha Faculty of Medicine Ethical Committee was the Institutional Review Board (IRB), from which we gained the ethical approval of this study protocol. Also, all parturients who participated in this trial singed written informed consent after careful counseling regards risks and benefits of bilateral total salpingectomies (BTS) and bilateral partial salpingectomies (BPS) as sterilizing procedures performed at the time of cesarean section (CS).

All parturients scheduled for CS either as electively or emergency procedures who requested or might request permanent sterilization were asked to participate in this study after careful counseling regarding permanent natures of this sterilizing procedures as well as the risks and benefits of this sterilizing procedures by the investigators of this trial (MAE and ANME). Eligibility criteria were termed : Pregnant women $\geq 38$ weeks gestational age with an adequate number of offsprings $\geq$ three child's of both sex, fit for surgery, i.e., American Society of Anesthesiologist (ASA) physical status I-II whom might be undergoing cesarean delivery due to any indications including a maternal request of sterilization. Exclusion criteria included women $<30$ years old, parturients at risk of cesarean hysterectomy, women with prior adnexal or ovarian surgical procedures and groups of women who may regret with desire for future fertility as well as women with family history of breast or ovarian cancer or who are known to be carriers for hereditary ovarian cancer syndromes as well as women who were at higher risk for potential surgical complications as women with immuno-compromising disease, chronic steroid user, on immunosuppressive therapy and women on therapeutic or prophylactic anticoagulation drugs.

Parturients were recruited sequentially and randomly allocated to receive either standard postpartum tubal ligation (STL) which is BPS according to our routine practice $($ control group $=$ BPS group $=$ STL group $)$ or bilateral total (complete) salpingectomies (intervention group $=$ BTS group $=\mathrm{BCS}$ group) at 1: 1 ratio. The trial Bio-statistician created a randomized treatment allocation scheme of 12 blocks of equal size of 10 participants by computer number generators and transferred the randomization scheme into sequentially numbered sealed opaque envelope containing either of allocation procedures. Six blocks were stored at El-Hayat Center ; two blocks were stored at BUH, while four blocks were taken by ANME to be conducted on his private patients. Participants were randomized at entering the operation room. After randomization, the investigators as well as participants were not blinded to group assignment anymore, while blinding was maintained on clinical pathologist as well as sonographer who later evaluated the participant's hormones and ovarian ultrasound status. Also, caregivers and data collectors were blinded to group 
assignment. CS was usually performed under regional anesthesia with or without sedation, usually through transverse skin incision as well as transverse uterine incision. After uterine incision closure, the obstetricians, always MAE or ANME, performed classic STL (BPS) in conventional way (control group), where loop of FT was caught, clamped, cut, doubly ligated, then both tubal ends were cauterized bilaterally or complete salpingectomy on either side (intervention group) was done with aids of bipolar clamp started at fimbrial side, taking care of injuring ovarian blood supply in the infundibulopelvic ligament, then cut the coagulated mesosalpinx immediately under the FT until reach the cornual ends, taking care of injuring the round or uter-ovarian ligaments.

Participants-related data were collected including all relevant history, surgical procedures including total operative time, tubal procedures time, total blood loss, tubal procedures related blood loss, type of anesthesia, CS techniques, additional procedures as adhesiolysis, all complication and adverse operative events as well as postoperative course until complete recovery. All women were contacted after one year by their primary obstetrician for evaluation of their ovarian reserve (OR), women who responded and decided to complete this study were asked to come within the first four days of menstrual flow and were evaluated by transvaginal ultrasound. Women who founded to have $>10 \mathrm{~mm}$ dominant follicle were asked to come back in next cycle; while women with quiescent ovaries were sent in the same day to the laboratory where they gave blood sample and sonographer where they were examined with transvaginal two and three-dimensional ultrasound (2 and 3DUS). Blood samples were collected from the participant's women via venipuncture, and they centrifuged the sample at $3500 \mathrm{rpm}$ for 10 minutes within 30 minutes to 4 hours maximally stored at $4^{\circ} \mathrm{C}$. Aliquots of serum samples were frozen at $-80^{\circ} \mathrm{C}$ till collection of all study samples were done in a single run. AMH was measured by Gen II-sensitive ELISA assay AMH kit (Beckman Coulter, Inc., Brea, CA, USA) with detection limits of $0.16 \mathrm{ng} / \mathrm{ml}$ to $10.4 \mathrm{ng} / \mathrm{ml}$ and inter-intra assays variation coefficients of $\leq 0.4 \%$ and $3.4 \%$ respectively. FSH was analyzed a CoBASe 411 auto-analyzer (Roche Diagnostics, Germany) by an ECLIA method with sensitivity limits of $0.100 \mathrm{Iu} / \mathrm{ml}$, while inter-intra assays variation coefficients were $\leq 3.5 \%, 2.6 \%$, respectively.

The same sonographer performed TV2 and 3DUS to all included women at cycle day 1-4, on voluson pro 370 (GE, health care ultrasound, Switzerland) assessing peak systolic velocity (PSV) of ovarian vessels on 2D colour Doppler mode then capture scan for both ovaries on 3D volumetric and $3 \mathrm{D}$ power Doppler mode evaluating the following items. Antral follicular count (AFC) as well as 3D vascularization indices ; namely, vascular index (VI), flow index (FI) and vascular flow index (VFI) with aids of sonography-based automated volume count and virtual organ computer-aided analysis imaging programs (SonoAVCTM and VOCA / TM, GE, health care ultrasound, Switzerland).

The main outcomes were differences between control group (BPS) and intervention group (BTS) regarding ovarian reserve parameters including $\mathrm{AMH}, \mathrm{FSH}, \mathrm{AFC}$, PSV, VI, FI, VFI as well as calculated ovarian age (OvAge/ year) from the following formula OVAge $=48.05-3.14$ $\mathrm{AMH}+0.07 \mathrm{FSH}-0.77 \mathrm{AFC}-0.11 \mathrm{FI}+0.25 \mathrm{VI}+0.1$ $\mathrm{AMH} \times \mathrm{AFC}+0.02 \mathrm{FSH} \times \mathrm{AFC}(24,25)$ at one year after performed tubal sterilizing procedure at time of cesarean delivery (CD). The subsidiary outcomes were related to operational feasibility as well as surgical consequences of tubal sterilizing procedures including operative time as well as total and tubal procedure related estimated blood loss, blood transfusion and related organs injuries.

A sample size of 45 per group were needed to detect a clinical meaning significant difference in serum $\mathrm{AMH}$ between control and intervention groups of $0.2 \mathrm{ng} / \mathrm{ml}$ as reported by van Rooij et al.(26) in age group between 36 - 40 years old of $-0.02(95 \%$ CI of $-0.3,-0.1)$ utilizing sample size APP for android(27) at study power of $80 \%$ as well as double-sided significance of $0.05 \%$ utilized the unpaired t-test. As we anticipate a large portion of loss to flow $(30 \%), 120$ women in total were included in this trial.

We followed intention to treat analysis (ITT), and we used SPSS version 25 (IBM, USA) to perform the statistical analysis. As our continuous variables were normally distributed we presented it as means \pm standard deviations (ranges) and we compared between them with independent student t-test, while we presented categorical variables as numbers and percentages and used Fisher's extract test to compare between them. To figure out significance, we used point estimate difference with $95 \%$ conference interval as well as setting $P$ value at $<0.05 \%$.

\section{INTRODUCTION}

We initially examined 200 parturients for eligibility in this study. 80 parturients were excluded for various reasons as presented in Figure (1). We allocated 120 eligible parturients to tubal sterilization via standard tubal ligation $(\mathrm{STL})$ (bilateral partial salpingectomies $(\mathrm{BPS}))($ no $=60)$ (control group) or via bilateral total salpingectomies (BTS) (intervention group (no $=60$ ). All randomized women received the allocated intervention or treatment, while we failed to follow up with ten women in the BTS group and 12 women in the BPS group. In total, 102 women were completely followed up at one-year post sterilization. The data of 120 parturients were available for surgical outcome analysis ; while only data of 102 women were available for ovarian reserve parameter outcomes analysis (Figure 1). 


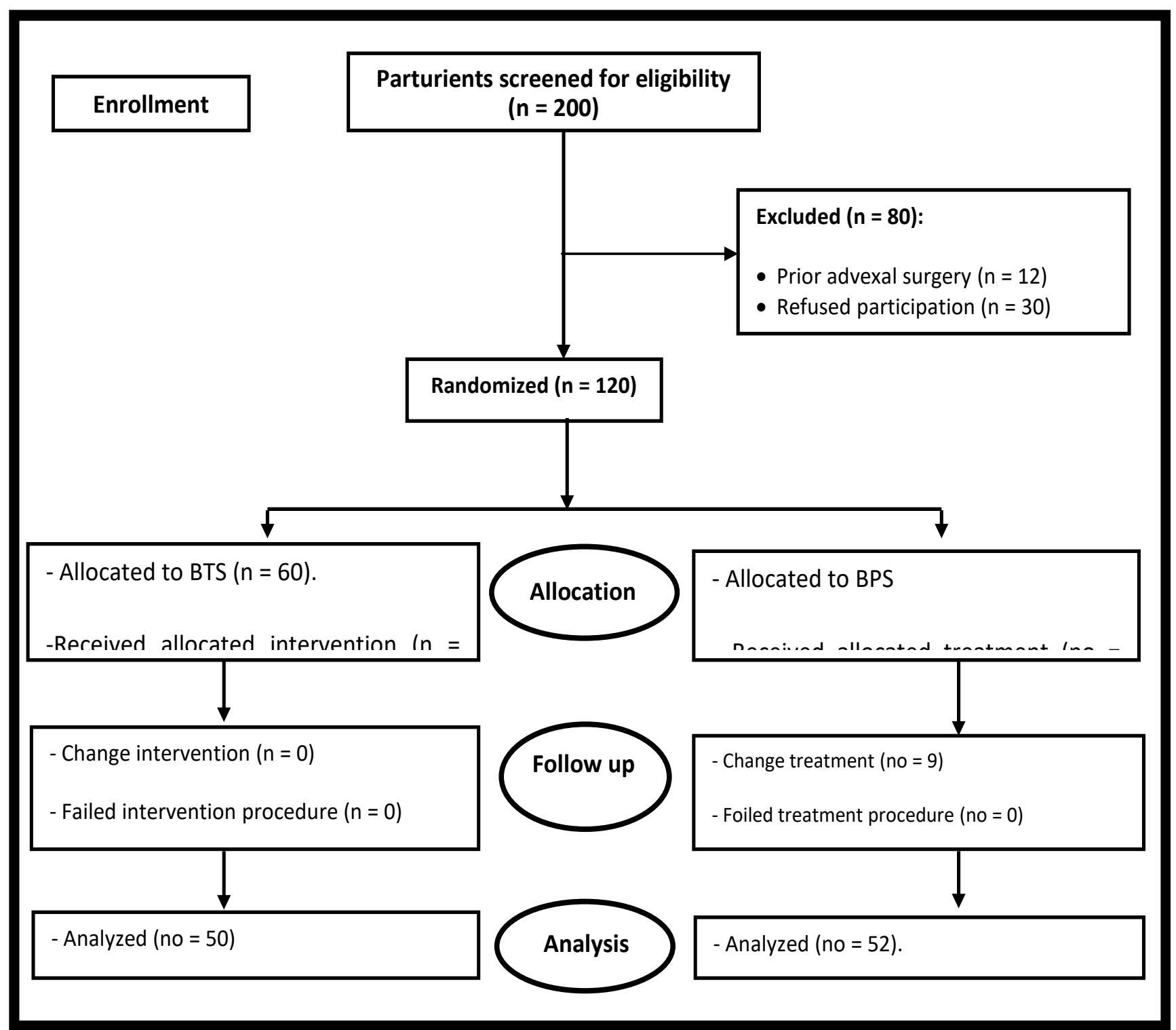

Fig. 1: CONSORT flow diagram of parturients undergoing BTS versus BPS included in STORE trial.

Table (1) displayed the trial cohort demographic, clinical, surgical and obstetrical characteristics and demonstrated that both groups didn't differ concerning these baseline criteria $(P>0.05)$.

Table (2) presented the ovarian reserve parameters differences at one year after performed tubal sterilization (TS) and demonstrated that there is no statistically significant difference between both groups regarding AMH $(P=0.30)$, FSH $(P=0.23)$, AFC $(P=0.40)$, ovarian volume $(P=0.88), \operatorname{PSV}(P=0.11), \mathrm{VI}(P=0.85), \mathrm{VFI}$
$(P=0.69)$, FI $(P=0.90)$ and OvAge $(P=0.90)$.

Table (3) presented the subsidiary outcomes of the STORE trial including operational feasibility as well as surgical consequences of both tubal sterilization procedures ; namely, BTS and BPS and showed that there were no significant differences between both groups $(P>0.05)$.

No apparent consequences were noticed in either group of the STORE cohort trial could be attributable to either received tubal sterilization procedures. 
Table 1: Baseline demographic, clinical, surgical and obstetrical criteria of parturients undergoing BTS and BPS included in STORE trial.

\begin{tabular}{|c|c|c|c|c|}
\hline Characteristic & $\begin{array}{l}\text { Intervention Group (BTS) } \\
\qquad(\mathrm{n}=60)\end{array}$ & $\begin{array}{l}\text { Control group (BPS) } \\
\qquad(\mathrm{n}=55)\end{array}$ & $\Delta(95 \%$ C.I $)$ & $P$ value \\
\hline Age $^{*}$ (year) & $35.81 \pm 5.62(28.17,42.23)$ & $36.21 \pm 6.21(28.21-43.20)$ & $-0.40(-1.74,2.54)$ & $=0.71$ \\
\hline $\mathrm{BMI}^{*}\left(\mathrm{~kg} / \mathrm{m}^{2}\right)$ & $31.62 \pm 6.91(24.21-38.21)$ & $32.21 \pm 7.21(23.82-40.21)$ & $-0.59(-254,3.72)$ & $=0.70$ \\
\hline Gravidity*(n) & $5.81 \pm 3.62(3-10)$ & $6.21 \pm 4.12(3-8)$ & $-0.46(-0.94,1.86)$ & $=0.51$ \\
\hline $\operatorname{Parity}^{*}(\mathrm{n})$ & $4.91 \pm 3.42(3-8)$ & $5.41 \pm 3.92(3-8)$ & $-0.50(-0.83,1.83)$ & $=0.45$ \\
\hline Number of living children*(n) & $3.62 \pm 2.81(3-6)$ & $3.92 \pm 3.21(3-5)$ & $-0.30(-0.79,13.9)$ & $=0.58$ \\
\hline Number of prior $\mathrm{CS}^{*}(\mathrm{n})$ & $3.62 \pm 2.81(0-6)$ & $3.83 \pm 2.91(0-6)$ & $-0.21(-0.82,1.24)$ & $=0.68$ \\
\hline \multicolumn{5}{|c|}{ Prior pelvic - abdominal surgery ${ }^{* *}$} \\
\hline None & $12(20 \%)$ & $13(21.6 \%)$ & $-1.6 \%(-12.94,16.07)$ & $=0.82$ \\
\hline $\mathrm{CS}$ & $36(63.4 \%)$ & $39(65 \%)$ & $-1.6 \%(-15.17,18.25)$ & $=0.85$ \\
\hline Other & $10(16.6 \%)$ & $8(13.4 \%)$ & $3.2 \%(-9.89,16.25)$ & $=0.62$ \\
\hline \multicolumn{5}{|l|}{ Co-Morbidities $^{* *}$} \\
\hline None & $48(70 \%)$ & $50(83.4 \%)$ & $-3.4 \%(-10.59,17.27)$ & $=0.63$ \\
\hline HTN & $4(6.7 \%)$ & $5(8.3 \%)$ & $-1.6 \%(-8.79,12.13)$ & $=0.74$ \\
\hline $\mathrm{DM}$ & $5(8.3 \%)$ & $3(5 \%)$ & $3.3 \%(-6.59,13.56)$ & $=0.47$ \\
\hline Other & $3(5 \%)$ & $2(3.3 \%)$ & $1.7 \%(-6.96,10.72)$ & $=0.64$ \\
\hline \multicolumn{5}{|l|}{ Type of CS ${ }^{* *}$} \\
\hline Primary & $14(23.3 \%)$ & $12(20 \%)$ & $3.3 \%(-11.75,17.90)$ & $=0.66$ \\
\hline Repeat & $46(76.7 \%)$ & $48(80 \%)$ & $-3.3 \%(-11.75,17.90)$ & $=0.66$ \\
\hline Gestational age* (weeks) & $38.82 \pm 1.23(38.21,40.92)$ & $38.91 \pm 1.24(38.31,41.21)$ & $-0.09(-0.35,0.53)$ & $=0.68$ \\
\hline \multicolumn{5}{|l|}{$\mathrm{ASA}^{* *}$} \\
\hline I & $44(73.3 \%)$ & $46(76.7 \%)$ & $-3.4 \%(-12.00,18.61)$ & $=0.66$ \\
\hline II & $16(26.7 \%)$ & $14(23.3 \%)$ & $3.4 \%(-12.00,18.61)$ & $=0.66$ \\
\hline \multicolumn{5}{|l|}{ Anesthesia type ${ }^{* *}$} \\
\hline Spinal & $42(70.0 \%)$ & $45(75 \%)$ & $5 \%(-10.86,20.54)$ & $=0.54$ \\
\hline Spinal with sedation & $16(26.7 \%)$ & $12(20 \%)$ & $6.7 \%(-8.45,21.50)$ & $=0.38$ \\
\hline General & $2(3.3 \%)$ & $3(5 \%)$ & $-1.7 \%(-6.96,10.72)$ & $=0.64$ \\
\hline Neonatal weight $(\mathrm{Kg})^{*}$ & $2.93 \pm 1.91(2.62-3.86)$ & $3.13 \pm 1.89(2.86,3.96)$ & $-0.2(-0.46,0.88)$ & $=0.56$ \\
\hline
\end{tabular}

Abbreviations: STORE: Tubal sterilization ovarian reserve assessment trial, BTS: bilateral total salpingectomies, BPS: bilateral partial salpingectomies, BMI: Body mass index, CS: Cesarean section, HTN: Hypertension, DM: Diabetus mellitus, ASA: American society of anesthesiologists physical status, $\Delta(95 \% \mathrm{CI})$ : Point estimate difference with $95 \%$ confidence interval.

- Values were given as mean \pm Standard deviation (range)* or number (percentage)**

$-P<0.05$ : Statistically significant. 
Table 2: Primary (ovarian reserve parameters) outcomes differences at one year between women undergoing BTs and BPS in STORE Trial.

\begin{tabular}{lcccc}
\hline Outcome & $\begin{array}{c}\text { Intervention group }(\mathrm{BTS}) \\
(\mathrm{n}=60)\end{array}$ & $\begin{array}{c}\text { Control group }(\mathrm{BPS}) \\
(\mathrm{n}=60)\end{array}$ & $\Delta(95 \%$ C.I $)$ & $P$ value \\
\hline $\mathrm{AMH}^{*}(\mathrm{ng} / \mathrm{ml})$ & $1.62 \pm 1.02(0.34-3.82)$ & $1.82 \pm 1.12(0.44-4.21)$ & $-1.02(-0.18,0.58)$ & $=0.30$ \\
$\mathrm{FSH}^{*}(\mathrm{mIu} / \mathrm{ml})$ & $8.16 \pm 4.26(4.28-15.61)$ & $9.21 \pm 5.36(5.62-18.61)$ & $-1.05(-0.70,2.80)$ & $=0.23$ \\
$\mathrm{AFC}^{*}(\mathrm{n})$ & $10.62 \pm 6.32(5-19)$ & $11.66 \pm 7.32(4-20)$ & $-1.04(-1.43,3.51)$ & $=0.40$ \\
$\mathrm{PSV}^{*}(\mathrm{~cm} / \mathrm{s})$ & $14.61 \pm 6.71(6.82-18.31)$ & $16.71 \pm 7.81(6.62-19.36)$ & $-2.10(-0.53,4.73)$ & $=0.11$ \\
$\mathrm{VI}^{*}(\%)$ & $1.32 \pm 2.63(0.32-3.88)$ & $1.23 \pm 2.21(0.42-3.86)$ & $0.08(-0.95,0.79)$ & $=0.85$ \\
$\mathrm{FI}^{*}(1-100)$ & $28.21 \pm 14.61(24.61-36.63)$ & $29.31 \pm 15.61(23.62-35.36)$ & $-1.10(-4.36,6.56)$ & $=0.69$ \\
$\mathrm{VFI}^{*}(1-100)$ & $1.02 \pm 1.66(0.78-1.22)$ & $0.98 \pm 1.86(0.72-1.13)$ & $0.04(-0.67,0.59)$ & $=0.90$ \\
OvAge $^{*}($ year $)$ & $36.82 \pm 12.63(30.61-40.22)$ & $37.12 \pm 13.62(30.22-40.62)$ & $-0.30(-4.44,5.04)$ & $=0.90$ \\
Ovarian volume $\left(\mathrm{cm}^{3}\right)$ & $5.63 \pm 6.32(3.36-10.62)$ & $5.82 \pm 8.43(3.12-11.63)$ & $-0.19(-2.50,2.88)$ & $=0.88$ \\
\hline
\end{tabular}

Abbreviations: BTS: bilateral total salpingectomies (intervention group), BPS: bilateral partial salpingectomies, (STL) (control group), STORE: Tubal sterilization ovarian reserve assessment trial, AMH: anti-müllerian hormone, FSH: follicular stimulating hormone, AFC: antral follicular count, PSV: peak systolic velocity, VI: vascular index, FI: flow index, VFI: vascular flow index, OvAge: calculated ovarian age, $\Delta(95 \% \mathrm{CI})$ : Point estimate difference with $95 \%$ confidence interval.

- Values were given as mean \pm Standard deviation (range)*.

$-P<0.05$ : Statistically significant.

Table 3: Ancillary (operational feasibility and consequences) outcomes differences between women undergoing BTS versus BPS in STORE trial.

\begin{tabular}{|c|c|c|c|c|}
\hline Outcome & $\begin{array}{l}\text { Intervention group (BTS) } \\
\qquad(\mathrm{n}=60)\end{array}$ & $\begin{array}{l}\text { Control group (BPS) } \\
\qquad(\mathrm{n}=60)\end{array}$ & $\Delta(95 \% \mathrm{CI})$ & $P$ value \\
\hline Surgical feasibility of $\mathrm{TS}^{* *}$ & $60(100 \%)$ & $60(100 \%)$ & $0.0 \%(-7.27,7.27)$ & $=1.00$ \\
\hline Total operative time* $(\mathrm{min})$ & $55.63 \pm 12.63(45-80)$ & $58.62 \pm 13.64(40-90)$ & $-2.99(-1.76,7.74)$ & $=0.21$ \\
\hline TS operative time ${ }^{*}(\mathrm{~min})$ & $12.61 \pm 8.61(8-15)$ & $13.25 \pm 9.62(6-15)$ & $-0.64(-2.66,3.94)$ & $=0.70$ \\
\hline Total operative blood loss ${ }^{*}(\mathrm{ml})$ & $750 \pm 150(600-1200)$ & $800 \pm 200(500-1300)$ & $-50.00(-13.91,113.91)$ & $=0.12$ \\
\hline TS operative blood loss*(ml) & $8.61 \pm 6.21(5-20)$ & $9.61 \pm 8.41(5-20)$ & $-1.00(-1.67,3.67)$ & $=0.46$ \\
\hline Change in hemoglobin* $(\mathrm{gm} / \mathrm{dl})$ & $0.98 \pm 0.86(0.66-1.62)$ & $0.95 \pm 0.89(0.77-1.53)$ & $0.03(-0.34,0.28)$ & $=0.85$ \\
\hline Blood transfusion ${ }^{* * a}$ & $1(1.6 \%)$ & $1(1.6 \%)$ & $0.0 \%(-7.27,7.27)$ & $=1.00$ \\
\hline Postoperative pain score*(VAS) & $6.91 \pm 5.61(3.6-8.9)$ & $6.71 \pm 5.33(3.8-9.2)$ & $0.20(-2.17,1.77)$ & $=0.84$ \\
\hline Intraoperative complications ${ }^{* * b}$ & $1(1.6 \%)$ & $1(1.6 \%)$ & $0.0 \%(-7.27,7.27)$ & $=1.00$ \\
\hline Postoperative complications ${ }^{* * c}$ & $10(16.6 \%)$ & $13(21.6 \%)$ & $-5.0 \%(-9.20,19.01)$ & $=0.48$ \\
\hline Hospital stay*(n) $^{*}$ & $10.50 \pm 8.36(6-48)$ & $11.56 \pm 9.42(6-48)$ & $-1.06(-2.15,4.27)$ & $=0.51$ \\
\hline
\end{tabular}

Abbreviations: BTS: bilateral total salpingectomies (intervention group), BPS: bilateral partial salpingectomies, (STL) (control group), STORE: Tubal sterilization ovarian reserve assessment trial, TS: tubal sterilization, VAS: Visual analogue scale score, $\Delta$ ( $95 \%$ CI): Point estimate difference with $95 \%$ confidence interval.

- Values were given as mean \pm Standard deviation (range)* or number (percentage)**

$-P<0.05$ : Statistically significant.

a) blood transfusion were postoperatively due to noticed moderate anemia

b) one case in each group of bladder injury.

c) Postoperative complications include persistent vomiting, paralytic ileus, urinary tract, and respiratory tract infections; all were managed successfully. 


\section{DISCUSSION}

Cesarean section is the most frequent operation performed in women worldwide and now in Egypt is considered to be an epidemic procedures, so this could be an opportunity to introduce the concept of bilateral total salpingectomies (BTS) as an effective sterilization procedure as well as ovarian cancer (OvCa) risk-reducing as a primary prevention in average-risk women for $\mathrm{OvCa}$, in parturients undergoing $\mathrm{CS}$ and requiring permanent sterilization. Bilateral partial salpingectomies (BPS) is the commonest performed surgical sterilization at CS as it is easy, safe as well as highly feasible ${ }^{[18,19,20]}$. BPS is found to be the most effective tubal sterilization with low ectopic rate $^{[13,14]}$ as well as might reduce the ovarian cancer risk especially type I endometrioid and clear cell carcinoma between $42 \%$ and $78 \%{ }^{[10,11,12]}$, however recent literatures introduce BTS as more effective superior procedure than BPS regarding contraceptive efficacy, eliminating risk of hydrosalpinges, pyosalpinges, adnexal accident, ectopics as well as effectively reducing OvCa especially type II high grade serous ovarian carcinomas (HGSOCs) as reviewed by Dilley et al. ${ }^{[6]}$. Also, theoretical modules evaluating the cost-effectiveness of RRS with hysterectomies, benign gynecology ${ }^{[8,29]}$, vaginal hysterectomies ${ }^{[30]}$ and cesarean deliveries ${ }^{[31,32]}$ proof the effectiveness of RRS. Computer models that investigate BPS versus BTS with CS deduced that both sterilization procedures are effective regarding ovarian cancer prevention and sterilization, despite that these models could not agree which procedure is more cost-effective than the other ${ }^{[31,32]}$. Subramanian et al. deduced that BTS is superior to BPS in their model, but Venkatesh et al. deduced that both procedures have similar cost-effectiveness. Despite that, there are a lot of recently published literatures demonstrating the theoretical throughs of the highest efficacy of BTS than BPS as cancer reducing as well as a sterilizing procedure ${ }^{[2,3,4,5,6,7,8]}$.

This prospective, randomized, concealed allocation, open-label, parallel group, superiority trial demonstrated that the impacts of BTS and BPS as sterilizing procedures at CS time on ovarian reserve parameters one year later including AMH, FSH, AFC, PSV, VI, FI, VFI, OvAge, and ovarian volume were similar $(P>0.05)$. Also, this study demonstrated that both procedures were equally feasible as well as perioperative surgical consequences were similar $(P>0.05)$. Studies were evaluating BTS versus BPS at the time of CS concentrating on feasibility as well as perioperative surgical consequences $^{[16,17,18,19,20,21,22]}$, while there is only one published RCT assessed procedures impacts on ovarian reserve(23). Researchers report a variable degree of feasibility, perioperative surgical consequences and this could be explained based on heterogeneity in surgical experiences as well as surgical techniques and surgical instruments. Studies evaluating ovarian reserve impacts of tubal sterilization did not show determinant changes on either hormonal or stromal blood supply ${ }^{[33,34]}$. Also, pilot RCT comparing 46 parturients (32 women of them completed the trial follow up, 16 in BTS arm and 18 BPS (STL)) found that AMH prior to CS as well as at $6-8$ weeks postpartum and the increase in $\mathrm{AMH}$ didn't differs significantly between both group $(\triangle \mathrm{AMH}$ were increase of $0.58 \pm 0.98$ in (BTS) vs. $0.39 \pm 0.41$ in (BPS) $\mathrm{ng} / \mathrm{ml}, \quad p=0.45)^{[23]}$. However, they reported prolonged operative time in BTS parturients over BPS participants by $13 \min (66.0 \pm 20.5$ versus $52.3 \pm 15.8$ minutes, $p=0.01$ ) as well as there were no differences in feasibility and surgical complications ${ }^{[23]}$. Studies regarding RRS in benign gynecology did not report detrimental impacts on ovarian reserve ${ }^{[35,36,37,38]}$.

Strengths include prospective nature, randomization, allocation concealment as well as power to : First, detect a prespecified clinical significant difference in an essential parameter of ovarian reserve. Second, evaluating ovarian reserve with a bunch of essential and recently introduce volumetric ultrasound parameters ; namely, $\mathrm{AMH}$, AFC with Sono AVC as well as vascularization indices with VOCAL. Third, assessing the ovarian reserve parameter one year after sterilization procedures away from conflicting effects of surgical procedures as well as pregnancy ${ }^{[39,40]}$. Fourth, investigating in one trial surgical consequences as well as impacts of sterilizing procedures on ovarian reserve and providing evidence regards the short and intermediate safety of BTS as an OvCa risk-reducing primary prevention procedure instead of BPS at time of CS in women completing their reproductive desire.

\section{LIMITATIONS OF THE STUDY}

Limitations include an inability to blind participants and investigators after allocation, however, the study outcomes were objective parameters unliable to be subjected to open-label nature of surgical trials. Second, underpowered of this trial to detect minor surgical consequences. Third assessing surrogate parameters of ovarian reserve rather than impacts of sterilizing procedures on the onset of menopausal symptoms itself. However, to study all these items, the trial might require a more significant number of participants as well as decades of follow up ${ }^{[41]}$, and this is behind our capabilities. Fourth, inability to evaluate the OvCa risk-reducing theoretical benefits of BTS over BPS, as evaluating such items requires a more significant number of participants as well as many decades of follow up ${ }^{[41]}$. Fifth, inability to generalize the results of the presented trial as its results were an individual experience of a senior obstetrician in his private practice, however, this could be an initiation for introducing this experience as well as providing such bipolar devices enabling completion of such procedures without complications. Sixth, lack of pathological examination of removed tubes in manner evaluating presences or absences of premalignant changes 
in the removed $\mathrm{FTs}^{[42]}$. We could recommend a more extended period of follow up to STORE participants cohort evaluating menopausal symptoms onset as well as ovarian reserve marker a few years later on as well as adnexal consequences of both sterilizing procedures and this trial-participant might be a nucleolus for a larger cohort evaluating ovarian cancer risk reduction by RRS.

\section{CONCLUSION}

The results of this RCT suggested that bilateral total salpingectomies (BTS) is a sterilizing procedure at time of cesarean delivery compared to bilateral partial salpingectomies (BPS) as standard tubal ligation procedures (STL) that don't have detrimental impacts on ovarian reserve parameters at one year after sterilization; as well as both procedures does not differ regard to surgical consequences including operative time when BTS performed with bipolar diathermy clamp. However, whether BTS or BPS might do better regard to menopausal symptoms onset and prevention of ovarian carcinomas as well as contraceptive effectiveness remain to be elucidated.

\section{CONFLICT OF INTEREST}

There are no conflicts of interest.

\section{ACKNOWLEDGMENT}

The authors want to thank their patients, fellows, data collectors, assistant staff including workers and nurses for helping them in completing this study.

\section{REFERENCES}

1. Lowe KA, Chia VM, Taylor A, O'Malley C, Kelsh M, Mohamed M, et al. An international assessment of ovarian cancer incidence and mortality. Gynecol. Oncol. 2013; 130 : 107 - 14.

2. Kurman RJ, Shih IeM. The origin and pathogenesis of epithelial ovarian cancer: a proposed unifying theory. Am J Surg Pathol 2010; 34: 433 - 43.

3. Erickson BK, Conner MG, Landen $\mathrm{CN}$ Jr. The role of the fallopian tube in the origin of ovarian cancer. Am. J. Obstet Gynecol. 2013; $209: 409-14$

4. Crum CP. Intercepting pelvic cancer in the distal fallopian tube: theories and realities. Mol Oncol. 2009; $3: 165$ - 70 .

5. Kindelberger DW, Lee Y, Miron A, Hirsch MS, Feltmate C, Medeiros $\mathrm{F}$, et al. Intraepithelial carcinoma of the fimbria and pelvic serous carcinoma: evidence for a causal relationship. Am J Surg Pathol. 2007; 31 : 161 - 9.

6. Dilley SE, Straughn JM Jr, Leath CA 3rd. The evolution of and evidence for opportunistic salpingectomy. Obstet Gynecol. 2017; $130: 8145$ - 24.

7. Walker JL, Powell CB, Chen L, Carter J, Bae Jump VL, Parker LP, et al. Society of Gynecology Oncology recommendations for the prevention of ovarian cancer. Cancer 2015; 121 : 2018-20.

8. Salpingectomy for ovarian cancer prevention. Committee Opinion. No. 620. American College of Obstetricians and Gynecologists. Obstet Gynecol 2015; $125: 279$ - 81 .

9. United Nations Department of Economic and Social Affairs, Population Division. World Contraceptive Patterns 2013. (Available at) http://www.un.org/ en/development/desa/population/publications/ family/contraceptive-wallchart-2013.shtml (Accessed September15, 2018).

10. Ely LK, Truong M. The role of opportunistic bilateral salpingectomy versus tubal occlusion or ligation for ovarian cancer prophylaxis. J Minim Invasive Gynecol. 2017; 24 : 371 -378.

11. Yoon SH, Kim SN, Shim SH, Kang SB, Lee SJ. Bilateral salpingectomy can reduce the risk of ovarian cancer in the general population: a meta-analysis. Eur J Cancer. 2016; 55 : 38 - 46.

12. Kwon JS, McAlpine JN, Hanley GE et al. Costs and benefits of opportunistic salpingectomy as an ovarian cancer prevention strategy. Obstet Gynecol. 2015; $125: 338-345$.

13. Peterson HB, Xia Z, Hughes JM, Wilcox LS, Tylor LR, Trussell J. The risk of pregnancy after tubal sterilization: findings from the U.S. Collaborative Review of Sterilization. Am J Obstet Gynecol., 1996; 174(4): 1161- 1168.

14. Peterson HB, Xia Z, Hughes JM, Wilcox LS, Tylor LR, Trussell J. For the U.S. collaborative review of Sterilization Working Group. The Risk of ectopic pregnancy after tubal sterilization. N Engl J Med., 1997; 336 : 762 - 767.

15. Rock JR, Jones HW. Tubal sterilization. Te Linde's operative gynecology, 10th ed. J.B. Lippincott, Philadelphia, 2008; 609 - 628.

16. Castellano T, Zerden M, Marsh L, Boggess K. Risks and Benefits of Salpingectomy at the time of sterilization. Obstetrical and Gynecological Survey 2017; 72: $663-8$. 
17. Lehn K, Gu L, Creinin MD, Chen MJ. Successful completion of total and partial salpingectomy at time of cesarean delivery. Contraception (2018).6.003

18. Danis R, Della Badia CR, Richard SD. Postpartum permanent Sterilization: could Bilateral salpingectomy replace bilateral tubal ligation? Journal of Minimally Invasive Gynecology 2016; 23: 928-32.

19. Shinar S, Blecher Y, Alpern S, Many A, Ashwal E, Amikam U, Cohen A. Total bilateral salpingectomy versus partial bilateral salpingectomy for permanent sterilization during cesarean delivery. Archives of gynecology and obstetrics 2017; 295: 1185 -9.

20. Hsieh G, Antony K, Masand R, Anderson M. A prospective feasibility study of postpartum distal salpingectomy. American College of Obstetricians and Gynecologists; 2014; Chicago.

21. Subramaniam A, Blanchard CT, Erickson BK, Szychowski J, Leath CA, Biggio JR, Huh WK. Feasibility of Complete salpingectomy compared with standard postpartum tubal ligation at cesarean delivery. A randomized controlled trial. Obstetrics and Gynecology, 2018; 132: 20-7.

22. Garcia C, Moskowitz OM, Chisholm CA, Duska LR, Warren AL, Lyons GR, Pettit KE. Salpingectomy compared with tubal ligation at cesarean delivery: A randomized controlled trial. Obstetrics and Gynecology 2018; 132: 29 - 34.

23. Garner Herman H, Gluck O, Keidar R, Kerner R, Kovo M, Levran D, Bar J, Sagiv R. Ovarian reserve following cesarean section with salpingectomy vs. tubal ligation: a randomized trial. American Journal of Obstetrics and Gynecology, 2017; 217: e1 - e6.

24. Venturella R, Lico D, Sarica A, Falbo MP, Gulletta E, Morelli M, et al. OvAge: a new methodology to quantify ovarian reserve combining clinical, biochemical and 3D-ultrasonographic parameters. J Ovarian Res. 2015; 8: 21.

25. Venturella R, Morelli M, Lico D, Cello AD, Rocca M, Sacchinelli A, Mocciaro R, D'Alessandro P, Maiorana A, Gizzo S and Zullo F. Wide excision of soft tissues adjacent to the ovary and fallopian tube does not impair the ovarian reserve in women undergoing prophylactic bilateral salpingectomy: results from a randomized, controlled trial. Fertil Steril. 2015 Nov; 104(5): 1332-9.

26. Van Rooij IAJ, Broekmans FJM, Scheffer GJ, Looman CWN, Habbema JDF, De Jong FH,
Fauser BJCM, Themmen APN, Te Velde ER. Serum antimullerian hormone levels best reflect the reproductive decline with age in normal women with proven fertility: a longitudinal study, Fertil. Steril. 83 (2005) 979987, http://dx.doi. org/10.1016/j.fertnstert.2004.11.029.

27. epiGenesys. A University of Sheffield Company. Available at : https : // www.epigenesys.org.uk/ portfolio/sampsize//

28. Known JS, McAlpine JN, Hanley GE, Finlayson SJ, Cohen T, Miller DM, et al. Costs and benefits of opportunistic salpingectomy as an ovarian cancer prevention strategy. Obstet Gynecol. 2015; $125: 338$ - 45.

29. Dilley SE, Havrilesky LJ, Bakkum-Gamez J, Cohn D, Michael Straughn J Jr, Caughey AB, et al. Cost -effectiveness of opportunistic salpingectomy for ovarian cancer prevention. Gynecol. Oncol. 2017; $146: 373$ - 9.

30. Cadish L, Shepherd JP, Barber EL, Ridgeway B. Risks and benefits of opportunistic salpingectomy during vaginal hysterectomy: a decision analysis. American Journal of Obstetrics and Gynecology, 2017; 217: 603.

31. Subramanian ABE, Blanchard CK, Erickson BK, Szychowski J, Leath CA, Biggio JR, Huh WK. The cost-effectiveness of salpingectomy versus standard tubal ligation at the time of cesarean delivery. Society for Maternal-Fetal Medicine, 38th Annual Meeting; Abstract \# 638; 2018; Dallas, TX.

32. Venkatesh KK, Clark LH, Stamilio DM. Costeffectiveness of opportunistic salpingectomy versus tubal ligation at the time of cesarean delivery, American Journal of Obstetrics and Gynecology; 2018, doi:10.1016/j.ajog.

33. Silva AL, Ré Cd, Dietrich C, Fuhrmeister IP, Pimentel A, Corleta HV. Impact of tubal ligation on ovarian reserve as measured by anti-Müllerian hormone levels: a prospective cohort study. Contraception, 2013; 88(6) : 70 .

34. 34 - Kelekci S, Yilmaz B, Yakut Y, Yasar L, Savan K. Sonmez SHormonal and ovarian stromal blood supply changes after laparoscopic tubal sterilization: a prospective controlled study. Contraception, 2006; 73(3) : $279-283$.

35. Morelli M, Venturella R, Mocciaro R, Di Cello A, Rania E, Lico D, Dalessandro P, Zullo F. Prophylactic salpingectomy in premenopausal low-risk women for ovarian cancer premium nonnocere, Gynecol. Oncol. 129 (2013) 448451. 
36. Findley AD, Siedhoff MT, Hobbs KA, Steege JF, Carey ET, McCall CA, Steiner AZ. Short-term effects of salpingectomy during laparoscopic hysterectomy on ovarian reserve: a pilot randomized controlled trial, Fertil. Steril. 100 (2013): 17041708.

37. Song T, Kim MM-LKMK, Jung YW, Yun BS, Seong SJ, Kwon S. Impact of opportunistic salpingectomy on anti-Mullerian hormone in patients undergoing laparoscopic hysterectomy: a multicentre randomized controlled trial, BJOG Int. J. Obstet. Gynaecol. (2016) 17, http://dx.doi. org/10.1111/1471-0528.14182.

38. Van Lieshout LAM, Pijlmanb B, Vosc MC, de GrootdMJM, Houtermane S, Corpus SFPJ, Harmsen MG, Vandenputa I, Pieka JMJ. Opportunistic salpingectomy in women undergoing hysterectomy: Results from the HYSTUB randomized controlled trial. Maturitas 107 (2018) : 1-6.
39. Venturella R, Lico D, Borelli M, Imbrogno MG, Cevenini G, Zupi E, Zullo F, Morelli M. 3 to 5 years later: long -term effects of prophylactic bilateral salpingectomy on ovarian function, J.Minim. Invasive Gynecol. 24 (2017): 145 -150.

40. Koninger A, kauth A, Schmidt B, Schmidt M, Yerlikaya G, kasimir-Bauer S, Kimming R, Birdir C. Anti-Mullerian - hormone levels during pregnancy and postpartum. Reproductive biology and endocrinology, 2013, $11: 60$.

41. Manchanda R, Menon U. Opportunistic bilateral salpingectomy (OBS) for the prevention of ovarian cancer should be offered in the context of a clinical trial. BJOG 2016;123:463.

42. Medeiros F, Muto MG, Lee Y, Elvin JA, Callahan MJ, Feltmate C, et al. The tubal fimbria is a preferred site for early adenocarcinoma in women with familial ovarian cancer syndrome. Am J Surg Pathol. 2006; $30: 230-6$. 